American Journal of Applied Sciences 6 (2): 313-316, 2009

ISSN 1546-9239

(C) 2009 Science Publications

\title{
Photoacoustic Measurement of Thermal Diffusivity of Polypyrrole Conducting Polymer Composite Films
}

\author{
${ }^{1}$ M.Y. Lim, ${ }^{1}$ W. Mahmood Mat Yunus, ${ }^{2}$ A. Kassim and ${ }^{3}$ H.N.M.E. Mahmud \\ ${ }^{1}$ Department of Physics, Faculty of Science, Universiti Putra Malaysia, \\ 43400 Serdang, Selangor, Malaysia \\ ${ }^{2}$ Department of Chemistry, Faculty of Science, University Putra Malaysia, \\ 43400 Serdang, Selangor, Malaysia \\ ${ }^{3}$ Faculty of Chemical Engineering, Universiti Teknologi MARA, \\ 40450 Shah Alam, Selangor, Malaysia
}

\begin{abstract}
The thermal diffusivity has been measured for the Polypyrrole-Polyethylene Glycol (PPyPEG) composite films by the open photoacoustic method. The experimental data were analyzed for thermal diffusivity by fitting procedure. The different process parameters such as different concentrations of insulating polymer, monomer and electrolyte and the different voltages used to prepare the PPy-PEG composite films brought changes in thermal diffusivity. The process condition of $0.20 \mathrm{M}$ pyrrole, $0.10 \mathrm{M}$ p-toluene sulfonate and $1 \times 10^{-3} \mathrm{M}$ PEG at 1.20 volt (vs SCE) appeared as the optimum condition and the PPy-PEG composite film produced from this optimum condition shows the highest thermal diffusivity $\left(7.88 \times 10^{-7} \mathrm{~m}^{2} \mathrm{~s}^{-1}\right)$.
\end{abstract}

Key words: Polypyrrole,polyethylene glycol, thermal diffusivity, photoacoustic method

\section{INTRODUCTION}

Recently, Photoacoustic spectroscopy (PAS) found important applications in research and analysis of inorganic, organic and biological solids and semisolid. It is a non-destructive technique that is applicable to almost all types of samples. It offers a minimal sample preparation and has the ability to look at opaque and scattering samples. In addition it has the capability to perform depth profiling experiments. PAS can be used for both qualitative and quantitative analysis. Photoacoustic is the production of acoustic waves by the absorption of light energy. The photoacoustic effect is based on the sensitive detection acoustic waves launched by the absorption of modulated laser radiation $^{[1-2]}$.

The PAS constitutes a simple, very reliable, experimental tool ${ }^{[3]}$ and has been widely used in measuring thermal diffusivity. The sensitivity tool of photoacoustic technique make this technique is suitable for studying the effect of synthesizing conditions in chemistry very often occurs in polymer synthesis ${ }^{[4-5]}$.

In our present study, the polypyrrole-polyethylene glycol (PPy-PEG) composite films were prepared by electrochemical method ${ }^{[6-7]}$.The main purpose of this study is to investigate the thermal diffusivity as a function of process parameters such as different concentrations of insulating polymer, monomer and electrolyte and the different applied voltages effects used to prepare PPy-PEG composite film.

\section{MATERIAL AND METHODS}

The aqueous solution in the one-compartment cell containing pyrrole monomer, p-toluene sulfonate electrolyte and the insulating polymer PEG, was electrochemically polymerized at a constant voltage (vs $\mathrm{SCE}$ ) at room temperature for $2 \mathrm{~h}$ to form PPy-PEG composite film. The composite film thus produced on the ITO glass surface as an insoluble film was rinsed thoroughly with distilled water and then peeled off from the electrode. It was then dried in the oven at $60^{\circ} \mathrm{C}$ for $24 \mathrm{~h}$.

Four series of PPy-PEG film samples were prepared by electrochemical polymerization technique. In the first series we fix the concentration of polypyrrole $(0.20 \mathrm{M}), \mathrm{p}$-toluene sulfonate $(0.10 \mathrm{M})$ and voltage (vs SCE) (1.2 volts) while the PEG was varied from $(0.5-9) \times 10^{-3} \mathrm{M}$. Theses samples were used to study the effect of PEG concentration on thermal diffusivity of PPy-PEG composite film. From electrical

Corresponding author: Mahmood, Department of Physics, Faculty of Science, Universiti Putra Malaysia, 43400 Serdang, Selangor, Malaysia Tel.: +6038946 6684 
Am. J. Applied Sci., 6 (2): 313-316, 2009

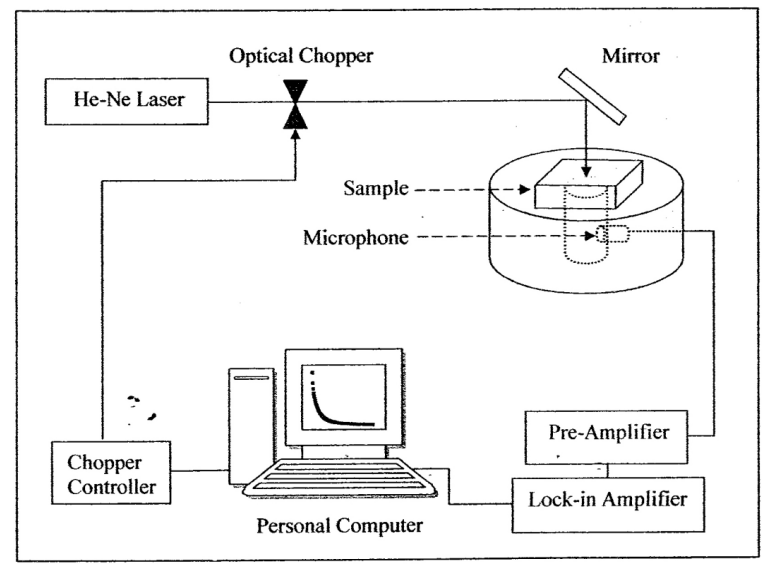

Fig. 1: Experiment setup for OPC detection technique

measurement ${ }^{[8]}$ it shown that the composite film with PEG concentration of $1 \times 10^{-3} \mathrm{M}$ gives the highest conductivity value. Therefore the second series of our sample were prepared at fix concentration, of PEG $\left(1 \times 10^{-3}\right) \mathrm{M}$ and let the concentration of polypyrrole change from (0.10-0.40) M. Again the electrical measurement shown that the sample with $0.20 \mathrm{M}$ concentration of polypyrrole gives the highest value of conductivity ${ }^{[8]}$. Furthermore we prepared the third series of PPy-PEG composite film by keeping the concentration of PEG and polypyrrole at $\left(1 \times 10^{-3}\right) \mathrm{M}$ and $0.20 \mathrm{M}$ respectively and change the concentration of p-toluene sulfonate from $0.05 \mathrm{M}-0.30 \mathrm{M}$. However in electrochemical polymerization technique the voltage (vs SCE) is one of the important parameter to produce a good uniformity film and to control the thickness of the sample. With this in mind we have prepared the forth series of PPy-PEG composite at different value of voltage (vs SCE).

Photoacoustic technique was used to measure thermal diffusivity of the prepared conducting composite films. Photoacoustic is the production of acoustic waves by the absorption of light. This effect is described as the periodic heating generated in the sample by a nonradiative deexcitation ${ }^{[9-11]}$ process due to the absorption of a modulated light source. In this study a heat transmission configuration known as open photoacoustic cell (OPC) was used. The sample was illuminated by the chopped laser beam which heats the surface and subsequently heats the gas in the cell. The periodic heating generates pressure variation in the surrounding gas medium. The experimental set up is shown in Fig. 1.

It has been shown that at low modulation frequency the photoacoustic signal for optically opaque samples is given by the expression ${ }^{[12]}$.

$$
S=\frac{A}{f} \exp (-b \sqrt{f})
$$

where, A is a constant and b is related to the thermal diffusivity of sample, $\alpha$ and given as

$$
\mathrm{b}=1_{\mathrm{s}} \sqrt{\pi / \alpha}
$$

By fitting the experimental data to the expression (1), the thermal diffusivity of the sample can be easily calculated.

\section{RESULTS AND DISCUSSION}

Figure 2 shows a typical photoacoustic signal as a function of modulated frequencies measured for frequency range of $5 \mathrm{~Hz}-100 \mathrm{~Hz}$. of PEG-Ppy composite sample prepared at 1.20 Volts (vs SCE). The solid line is the calculated values obtained by using Eq. (1). In this case the fitting results thermal diffusivity of PPy-PEG sample as $7.88 \times 10^{-7} \mathrm{~m}^{2} \mathrm{~s}^{-1}$. The same thermal diffusivity evaluation procedure has been implied to all of our samples. The results are shown in Fig. 3-6.

The PPy-PEG composite films prepared at various concentrations of PEG ranging from $0.5 \times 10^{-3} \mathrm{M}-9 \times 10^{-3}$ $\mathrm{M}$ in the aqueous solution containing $0.20 \mathrm{M}$ pyrrole and $0.10 \mathrm{M}$ p-toluene sulfonate at a potential of 1.20 volt (vs SCE) has been studied to investigate the effect of PEG concentration on the thermal diffusivity the films. Fig. 3. shows the thermal diffusivity increases from $3.87 \times 10^{-7} \mathrm{~m}^{2} \mathrm{~s}^{-1}-7.88 \times 10^{-7} \mathrm{~m}^{2} \mathrm{~s}^{-1}$ as the PEG concentration in the pyrrole solution increases up to $1 \times 10^{-3} \mathrm{M}$. This indicates that electron migration is an addition process in thermal transport properties, However at higher concentration of PEG it shows a decreasing trend from $7.88 \times 10^{-7} \mathrm{~m}^{2} \mathrm{~s}^{-1}$ $4.86 \times 10^{-7} \mathrm{~m}^{2} \mathrm{~s}^{-1}$ with further increase of PEG concentration from $1 \times 10^{-3} \mathrm{M}-9 \times 10^{-3} \mathrm{M}$. This is in a good agreement with the result of other polypyrrole composite films reported by other researcher ${ }^{[12]}$.

In the second series of samples we investigate the effect of pyrrole concentration on thermal diffusivity of PPy-PEG composite film. The pyrrole concentration has been changed from $0.10 \mathrm{M}-0.40 \mathrm{M}$, with p-toluene sulfonate dopant of $1 \times 10^{-3} \mathrm{M}$ of PEG respectively. The film was prepared at voltage of 1.20 Volt (vs SCE). Fig. 4. shows the thermal diffusivity values as a function of pyrrole concentration. A similar trend was also observed where the higher value of thermal diffusivity $\left(7.88 \times 10^{-7} \mathrm{~m}^{2} \mathrm{~s}^{-1}\right)$ was obtained for $0.20 \mathrm{M}$ of pyrrole concentration. The increase in thermal diffusivity at low 


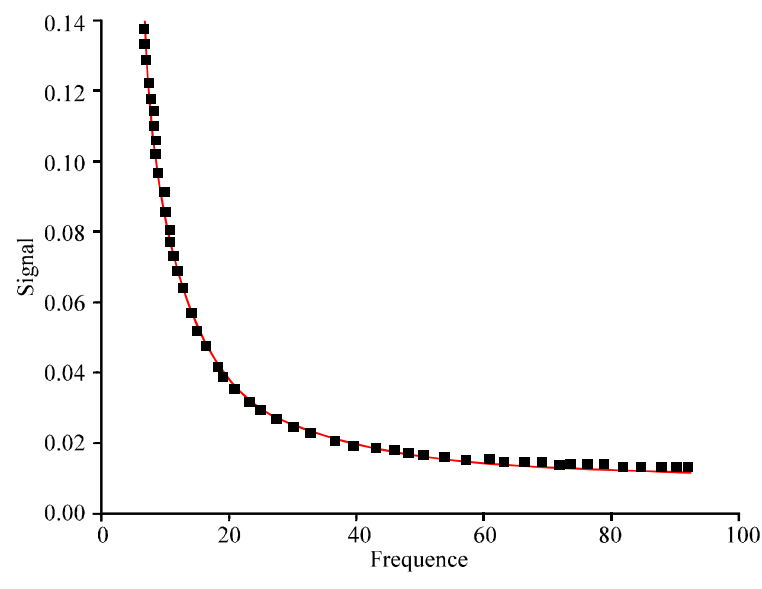

Fig. 2: Signal fitting for PPy-PEG Composite film

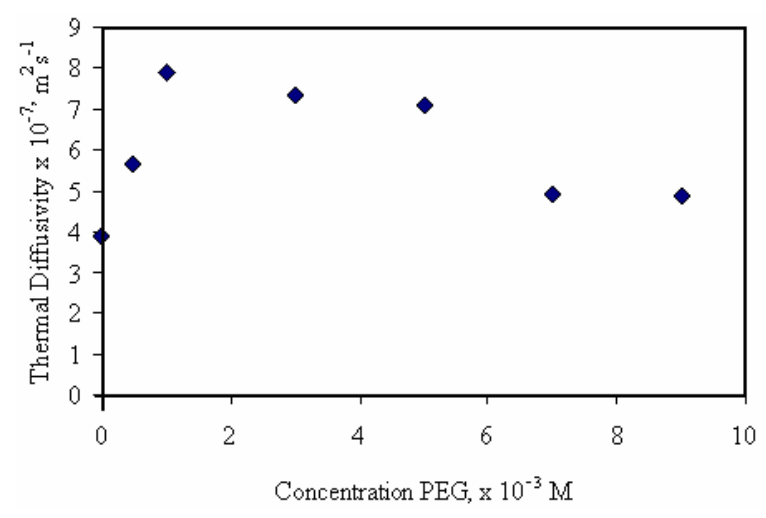

Fig. 3: Thermal diffusivity of PPy-PEG composite films versus PEG concentration

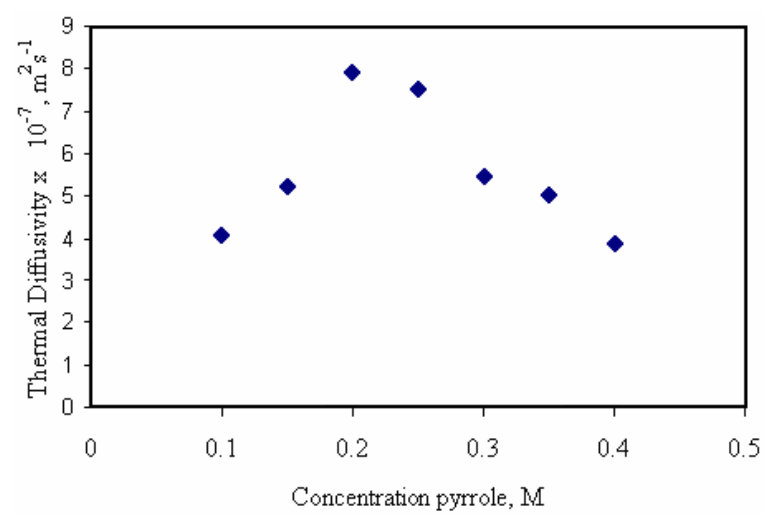

Fig. 4: Thermal diffusivity of PPy-PEG composite films versus pyrrole concentration

concentration of pyrrole $(<0.20 \mathrm{M})$ may due to the fact that the polymerization rate becomes too fast causing the increase of electron migration.

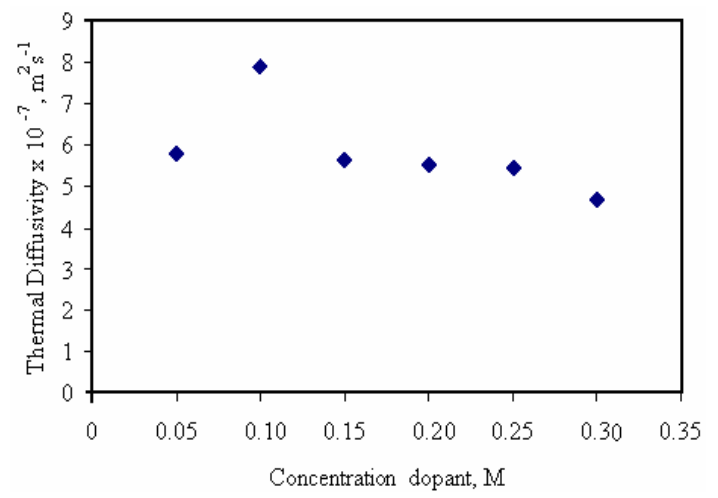

Fig. 5: Thermal diffusivity of PPy-PEG composite films against different p-toluene sulfonate concentration

To investigate the effect of dopant concentration on the thermal diffusivity of PPy-PEG composite films, we have prepared the samples with the concentration of $\mathrm{p}$ toluene sulfonate from $0.05-0.30 \mathrm{M}$. In presence of 0.20 $\mathrm{M}$ pyrrole, $1 \times 10^{-3} \mathrm{M}$ PEG and the voltage (vs SCE) was kept constant at 1.20 volts (vs SCE) for the whole samples in this series. The thermal diffusivity as a function of dopant concentration of PPy-PEG composite film prepared at different concentration of $\mathrm{p}$ toluene sulfonate is shown in Fig. 5. In this case the highest $\left(7.88 \times 10^{-7} \mathrm{~m}^{2} \mathrm{~s}^{-1}\right)$ value of thermal diffusivity was obtained for $0.10 \mathrm{M}$ of dopant concentration in PPy-PEG composite film. For concentration higher than $0.10 \mathrm{M}$, the thermal diffusivity decreases with the increasing of dopant concentration. The higher thermal diffusivity obtained for PPy-PEG film prepared from $0.10 \mathrm{M}$ p-toluene sulfonate may due to higher conjugation length which related to the electron migration process.

Since the applied potential plays an important role in electrochemical polymerization technique, we have prepared the PPy-PEG composite film by changing the potential from $0.40-1.50$ volts (vs SCE). For this last series of samples we kept the pyrrole solution, ptoluene sulfonate and PEG at the concentration of 0.20 $\mathrm{M}, 0.10 \mathrm{M}$ and $1 \times 10^{-3} \mathrm{M}$ respectively. Fig. 6 . shows the thermal diffusivity of the PPy-PEG composite films obtained at different potential (vs SCE). Obviously, the sample prepared at voltage of 1.20 volts (vs SCE) gives the higher thermal diffusivity $\left(7.88 \times 10^{-7} \mathrm{~m}^{2} \mathrm{~s}^{-1}\right)$. This can be explained by the fact that the increasing in applied voltage, the conjugation chain length (number of pyrrole monomer units) of PPy increases causing the increase in thermal diffusivity. This decrease in thermal diffusivity at the potential above 1.20 volt (vs SCE) may be due to overoxidation process in the sample. 


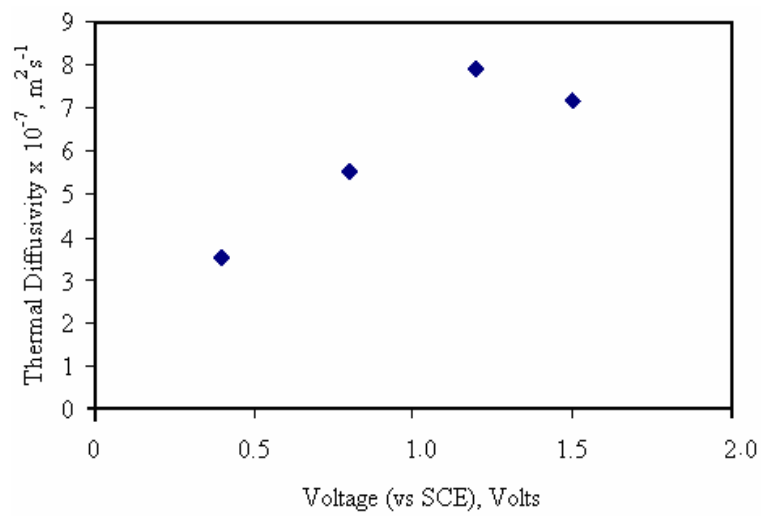

Fig. 6: Thermal Diffusivity of PPy-PEG composite films against different applied voltage (vs SCE)

\section{CONCLUSION}

Thermal diffusivity of PPy-PEG composite films prepared by electropolymerization was investigated using open photoacoustic technique. The PPy-PEG composite films prepared at $0.20 \mathrm{M}$ pyrrole monomer, $0.10 \mathrm{M}$ p-toluene sulfonate dopant and $1 \times 10^{-3} \mathrm{M}$ PEG at 1.20 volt (vs $\mathrm{SCE}$ ) gives the highest thermal diffusivity $\left(7.88 \times 10^{-7} \mathrm{~m}^{2} \mathrm{~s}^{-1}\right)$.

\section{ACKNOWLEDGEMENT}

The authors would like to thank the Malaysia Government for financial support. The laboratory facilities provided by the Department of Physics, UPM are also acknowledged.

\section{REFERENCES}

1. Lu. Y. 1988. Solitons and Polaron in Conducting Polymer. World Scientific, New Jersey, pp:74.

2. Heeger, J., S. Kivelson, J. R. Schriefer and W. P. Su. 1988. Solitons in conducting polymers. Mod. Phys.,60: 781-850.
3. Adams, M. J. and G. F. Kirkbright. 1977. Analytical optoacoustics spectrometry: the optoacoustic effect and thermal diffusivity, Analyst., 102: 281-292

4. Cesar, C. L., H. Vargas, F. Mendes and L. C. M. Miranda. 1983. Photoacoustic determination of thermal diffusivity of solids: Appl. to Cds. Appl. Phys. Lett., 43: 555-557.

5. Mansanares, A. M., A. C. Bento, H. Vargas, N. F. Leite and L. C. M. Miranda. 1990. Photoacoustic measurement of the thermal-properties of 2-layer system. Phys. Rev.B., 42: 4477-4486.

6. Diaz, F., K. K. Kanazawa and G. P. Gardini. 1979. Electrocjemical polymerization of pyrrole. J. Cham. Soc. Chem. Commun., 635-636.

7. Kanazawa, K. K., A. F. Diaz, R. H. Geiss, W. D. Gill, J. F. Kwak, J. A. Logan and J. F. Rabolt. 1979. Organic metals: Polypyrrole a stable synthetic 'Metallic' polymer. J. Chem. Soc. Chem. Commun., 854.

8. Lim, M. Y., H.N.M. E. Mahmud, A. Kassim and W. M. M. Yunus. 2007. Polypyrrole-polyethylene glycol conducting polymer composite films: Preparation and characterization. Synthetic Metals., 157:386-389

9. Toyoda, T. and T. Obikawa. 1966. Photoacoustic spectroscopy of $\mathrm{Cr}^{3+}$ in Ceramic $\mathrm{Al}_{2} \mathrm{O}_{3}$. Mater. Sci. Eng., B41:72-75.

10. Pinto, N., A. H. Vargas, N. F. Leite and L. C. M. Miranda. 1989. Photoacoustic investigation of semiconductor: Influence of carrier diffusion and recombination in $\mathrm{PbYe}$ and Si. Phys. Rev. N., 40:3924-3930.

11. Lima, C. A. S., M. B. S. Lima, L. C. M. Miranda, J. Baeza, J. Freer, N. Reyes, J. Ruiz and M. D. Silva. 2000. Photoacoustic characterization of bleached wood pulp and finished papers. Meas. Sci. Technol., 11:504-508.

12. Naoto, T., I. Satosh and K. Tsuyoshi. 1994. Thermal Diffusivity and Electrical Conductivity of Electropolymerized Polypyrrole Films. J. Poly. Sci. 32: 1899-1906. 